\title{
Numerical modelling the ultra-broadband mid-infrared supercontinuum generation in the As2Se3 photonic crystal fiber
}

\author{
Zheng Yan, Jianguo Wen, Chujun Zhao,Wenlong Wang,Yunlin Wang \\ Department of Information science and Engineering, Hunan University, Changsha, 10532, China
}

Keywords: Photonic crystal fiber, mid-infrared supercontinuum, ultra-broadband

\begin{abstract}
In this paper, ultra-broadband mid-infrared (MIR) supercontinuum (SC) generation ranging from 2 to $15 \mu \mathrm{m}$ in $\mathrm{As}_{2} \mathrm{Se}_{3}$ photonic crystal fiber (PCF) was investigated numerically by multipole method and the adaptive split-step Fourier method. By choosing different hole sizes and the pitch parameters of the $\mathrm{As}_{2} \mathrm{Se}_{3} \mathrm{PCF}$, the optimized nonlinear coefficients and dispersion profile of the fiber in the mid-infrared regime can be obtained. With the ultrafast pump laser centered around $4.3 \mu \mathrm{m}$, the broadband SC from 2 to $15 \mu \mathrm{m}$ can be generated. In the normal dispersion regime, the flatness of the SC can be improved. Moreover, the pulse durations of the pump laser have positive influence on the spectrum broadening as well as the pump peak power.
\end{abstract}

\section{Introduction}

Supercontinuum (SC) generation has found a large number of applications in diverse disciplines such as optical frequency metrology, spectroscopy astronomy, optical tomography, and infrared imaging [1, 2]. Usually, the SC can cover the visible and near-infrared spectrum [3, 4]. However, many applications need the spectrum could be broadened further towards mid-infrared region. Furthermore, the SC spectrum needs to be very broad and flat. Among the SC sources, fiber generated SC light provides a useful balance of brightness, coherence and bandwidth making it an appealing optical source for investigations in this spectral region [5]. In the late 1990s, the advent of PCF attracted widespread interest, and it led to revolution in the generation of SC [6]. Now silica fibers are widely used for SC generation, but the extending of the SC is limited around the wavelength of $2 \mu \mathrm{m}$ due to the material losses. Soft-glass fibers with low loss in mid-infrared regime have been considered as ideal candidates for development of broadband MIR SC sources [7-9]. Combined with the flexible dispersion characteristics of PCF and the low loss soft-glass, the MIR SC can be generated with appropriate pump sources [10].

Unlike all other soft glasses, chalcogenide glasses, especially As2Se3 and As2S3, have a larger refractive index and a higher nonlinear index, resulting in a greater modal confinement and a higher nonlinearity [11-13]. Moreover, they are transparent at a mid-IR wavelength around $10 \mu \mathrm{m}$. Recently, Shaw et al. have reported experimental work that demonstrates SC generation from 2.1 to $3.2 \mu \mathrm{m}$ in an As2Se3-based chalcogenide PCF with one ring of air holes in a hexagonal structure [13]; Price et al. have shown theoretically that it is possible to generate a mid-IR SC from 2 to $5 \mu \mathrm{m}$ using a bismuth-glass PCF [14]; Rim et al. found the SC up to $8 \mu \mathrm{m}$ in As2Se3 PCF with different pitches [12]; Wu obtained an output spectrum of 2-10 $\mu \mathrm{m}$ in As2Se3 PCF by using a $4 \mu \mathrm{m}$ pump [10]; Wang et al.obtained a mid-infrared broadband polarized SC up to $14 \mu \mathrm{m}$ in As2Se3 PCF [15].

In this paper, the appropriate structure of As2Se3 PCF with ZDW located nearby $4.5 \mu \mathrm{m}$ was designed. By comparing the spectrum for different relative hole size ( $d / \Lambda=0.35,0.37,0.4$ ) with hole-to-hole spacing $\Lambda=2 \mu \mathrm{m}$, we demonstrate that a broadband MIR SC output from 2 to $15 \mu \mathrm{m}$ can be generated with pump wavelength centered at $4.3 \mu \mathrm{m}$. By adjusting the parameters of the PCF and input laser, the extending range and the flatness of spectrum can be improved.

\section{Fiber design and theoretical model}

By using the multipole method, we can calculate and analyze the PCF. In our simulation, the relative hole size of $\mathrm{d} / \Lambda=0.35, \mathrm{~d} / \Lambda=0.37$, and $\mathrm{d} / \Lambda=0.4$ were chosen with hole-to-hole spacing $\Lambda=2$ $\mu \mathrm{m}$. In order to reduce loss, a short fiber length is used. For the refractive index of As2Se3, the 
Sellmeier equation was used as follows [10]:

$$
\begin{aligned}
& n(\lambda)=\left[1+\lambda^{2}\left[\mathrm{~A}_{0}{ }^{2} /\left(\lambda^{2}-\mathrm{A}_{1}{ }^{2}\right)+\right.\right. \\
& \left.\left.\mathrm{A}_{2}{ }^{2} /\left(\lambda^{2}-19^{2}\right)+\mathrm{A}_{3}{ }^{2} /\left(\lambda^{2}-4 \times \mathrm{A}_{1}{ }^{2}\right)\right]\right]^{0.5}
\end{aligned}
$$

where $\mathrm{A}_{0}=2.234921, \mathrm{~A}_{1}=0.24164, \mathrm{~A}_{2}=0.347441, \mathrm{~A}_{3}=1.308575$. Fig. 1 shows the calculated dispersion curve of designed PCF through the formula (2), which includes both waveguide and material dispersion.

$$
D=-\frac{\lambda}{c} \frac{d^{2} n}{d \lambda^{2}}
$$

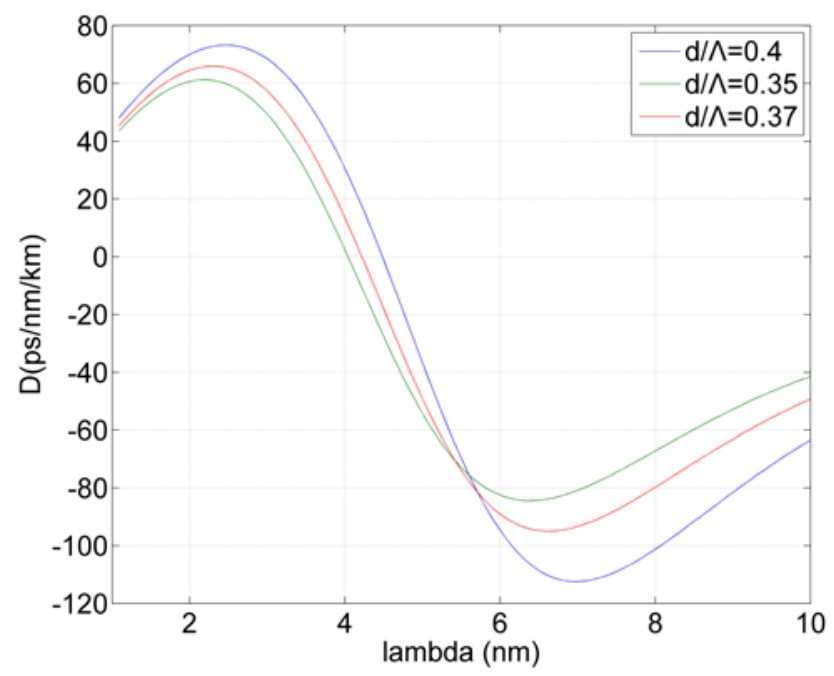

Fig. 1 The dispersion curve of $\mathrm{As}_{2} \mathrm{Se}_{3}$ PCF with different relative hole size and hole-to-hole spacing

$$
\Lambda=2 \mu \mathrm{m} \text {. }
$$

The SC generation can be described by nonlinear-Schrodinger equation (GNLSE):

$$
\frac{\partial A}{\partial z}+\frac{\alpha}{2} A-i \sum_{k \geq 1} \frac{i^{k} \beta_{k}}{k !} \frac{\partial^{k} A}{\partial t_{k}}=i \gamma\left[1+\frac{i}{\omega_{0}} \frac{\partial}{\partial t}\right]\left[\mathrm{A}(\mathrm{z}, \mathrm{t}) \int_{-\infty}^{\infty} R\left(\mathrm{t}^{\prime}\right)\left|A\left(\mathrm{z}, \mathrm{t}-\mathrm{t}^{\prime}\right)\right|^{2} \mathrm{dt} \mathrm{t}^{\prime}\right]
$$

where $A(z, t)$ is the complex amplitude of the light field, $\alpha$ is the fiber loss, $\beta_{k}$ is coefficient of high frequency $\omega_{0}$. The right-side item of Eq. (3) represents nonlinear optical effect, including self-phase modulation (SPM), four-wave mixing (FWM), stimulated Raman scattering (SRS), and etc. Raman response function can be written as :

$$
\begin{gathered}
h_{R}(\mathrm{t})=f_{a} \tau_{1}\left(\tau_{1}^{-2}+\tau_{2}^{-2}\right) \exp \left(-\tau / \tau_{2}\right) \times \sin \left(\tau / \tau_{1}\right)+ \\
f_{b} \tau_{3}\left(\tau_{3}^{-2}+\tau_{4}{ }^{-2}\right) \times \exp \left(-\tau / \tau_{4}\right) \sin \left(\tau / \tau_{3}\right) \\
R(\mathrm{t})=\left(1-f_{R}\right) \delta(\mathrm{t})+f_{R} h_{R}(\mathrm{t})
\end{gathered}
$$

In the simulation, $\quad \mathrm{fa}=0.7, \mathrm{fb}=0.3, \tau 1=23 \times 10-15, \tau 2=230 \times 10-15, \tau 3=20.5 \times 10-15, \tau 4=260 \times$ 10-15, $\mathrm{fR}=0.1$. The nonlinear coefficient can be expressed as $\gamma=n_{2} \omega_{0} / c A_{\text {eff }}=2 \pi n_{2} / \lambda A_{\text {eff }}$. Here, the calculated nonlinear coefficient is about $\gamma=3497 \mathrm{~km}-1 \mathrm{~W}-1$, which is much greater than the value of silica photonic crystal fiber. Through the split-step Fourier method, the process of pulse transmission can be simulated [10].

\section{Numerical simulation and analysis}

\subsection{The SC generation}

Because of the combined effects of nonlinear effects and dispersion, the spectrum will be expanded in the As2Se3 PCF. With the pump laser with duration $500 \mathrm{fs}$, wavelength centered at 4.3 $\mu \mathrm{m}$, and peak power $3 \mathrm{~kW}$, the simulated spectrograms for different propagation distance along the fiber can be obtained. By illuminating the pump into PCF with different dispersion profiles, the 
broadened spectrum is quite different. For the PCF with $\mathrm{d} / \Lambda=0.35$, the pump laser locates in the normal dispersion region, while for the PCF with $d / \Lambda=0.4$ the pump laser is in the anomalous dispersion region. As shown in Fig. 2, the spectral broadening can be divided into three stages, i.e. initial broadening, broadening, and saturated broadening. The initial broadening is mainly due to the SPM, the spectrum broadens symmetrically. When the transmission distance $\mathrm{L}=0.1 \mathrm{~cm}$, the broadening for the case in anomalous dispersion region is better than that of the normal dispersion region, which can be explained as follows. For the case with pump laser in the normal dispersion region of PCF, the input pulse laser broadens quickly in time-domain, which results in the peak power decreases and the nonlinear effect becomes weak, as shown in Fig. 2 (b). With increasing propagation distance greater than $0.5 \mathrm{~cm}$, the spread spectrum is tending to be saturated. It also can be seen that Fig. 2 (a) has a better flatness than Fig. 2 (b) for the short-wavelength side. It is mainly because that the spectral broadening on the short-wavelength is mainly due to the soliton fission and the Raman soliton self-frequency shift, the spectrum in Fig. 2 (b) will be complex, and the flatness will be low.

(a)

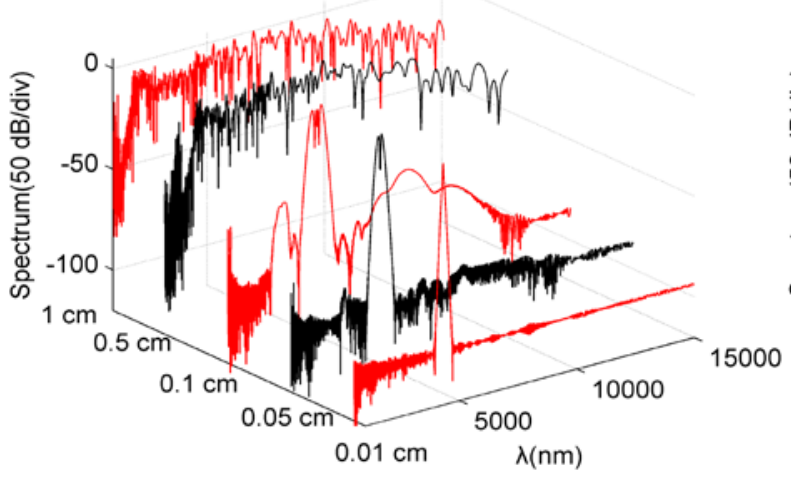

(b)

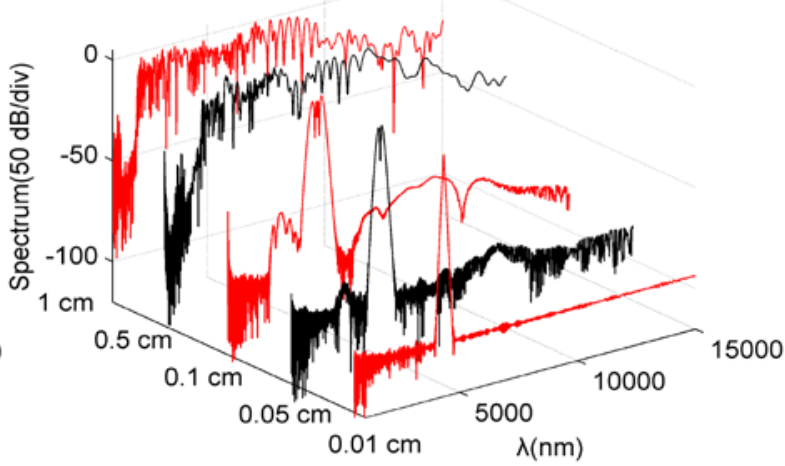

Fig. 2 (a) The SC spectra of PCF $(\Lambda=2$ and relative hole size $d / \Lambda=0.35)$ in different transmission distance. (b) The SC spectra of PCF $(\Lambda=2$ and relative hole size $d / \Lambda=0.4)$ in different transmission distance.

\subsection{The influence of PCF parameters on SC generation}

The curves in Fig. 3 show the corresponding simulated SC spectra output from a $1 \mathrm{~cm}$ long As2Se3 PCF fiber pumped by a $4.3 \mu \mathrm{m}$ femtosecond laser with pulse duration 500 fs and peak power $1 \mathrm{~kW}$ for $\mathrm{d} / \Lambda=0.35,0.37$, and 0.4 . We can find that, a continuum extending from 2000-15000 nm can be generated. For the material losses will be high when long-wavelength above $9 \mu \mathrm{m}$. We choose the short PCFs to avoid the large loss. When pumping in the normal regime for $\mathrm{d} / \Lambda=0.35$, the spectral broadening is the largest.

For the largest spectral broadening, the suitable structure of fiber and parameters of pump (wavelength, pulse-width, peak power) becomes very important. The As2Se3 PCF we chose has high nonlinear coefficient. It can guarantee the majority of the spectrum broadening occurred in the first few centimeters, and this will reduce the loss of the fiber. Through the simulation, we got spectrum broadening to reach $15 \mu \mathrm{m}$. Although Fig. 3 shows that the loss became large after $9 \mu \mathrm{m}$, as the power increases, spectral red shift will be obvious, so the spectrum is better when the peak power was increased to $3 \mathrm{~kW}$ 。 


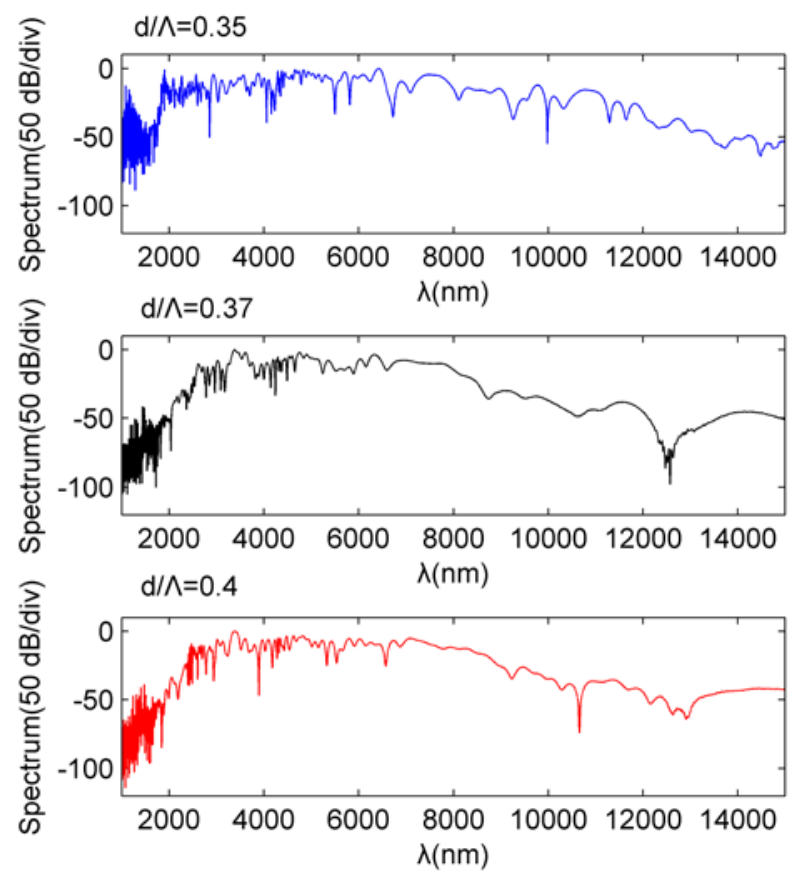

Fig. 3 Spectra of the SC generation with different relative hole size

3.3 The influence of input parameters

In order to optimize the continuous spectrum broadening, we further studied the influence of input parameters in As2Se3 PCF. Compared with different pulse width of the Gaussian pulse, their broadening was nearly unchanged. As Fig. 4 shown, when the peak power is constant and the pulse width is $800 \mathrm{fs,} \mathrm{broadening} \mathrm{reaches} \mathrm{the} \mathrm{largest.} \mathrm{The} \mathrm{simulation} \mathrm{results} \mathrm{illustrated} \mathrm{in} \mathrm{Fig.} 4$ suggest that, for a peak power beyond $3 \mathrm{~kW}$, a similar broadband MIR SC generation with the bandwidth than $10 \mu \mathrm{m}$ can be achieved. The asymmetry in the spectral power density of the short and long wavelength regions arises due to the different continuum generation mechanisms responsible for each side. The long wavelength side of the spectrum experiences additional gain due to stimulated Raman scattering, which transfers power from shorter to longer wavelengths. On the other hand, wavelengths below the pump is generated primarily by phase matched FWM. The flatness nature of the continuum can be attributed to the ensemble average of spectra produced by multiple solitons [12].
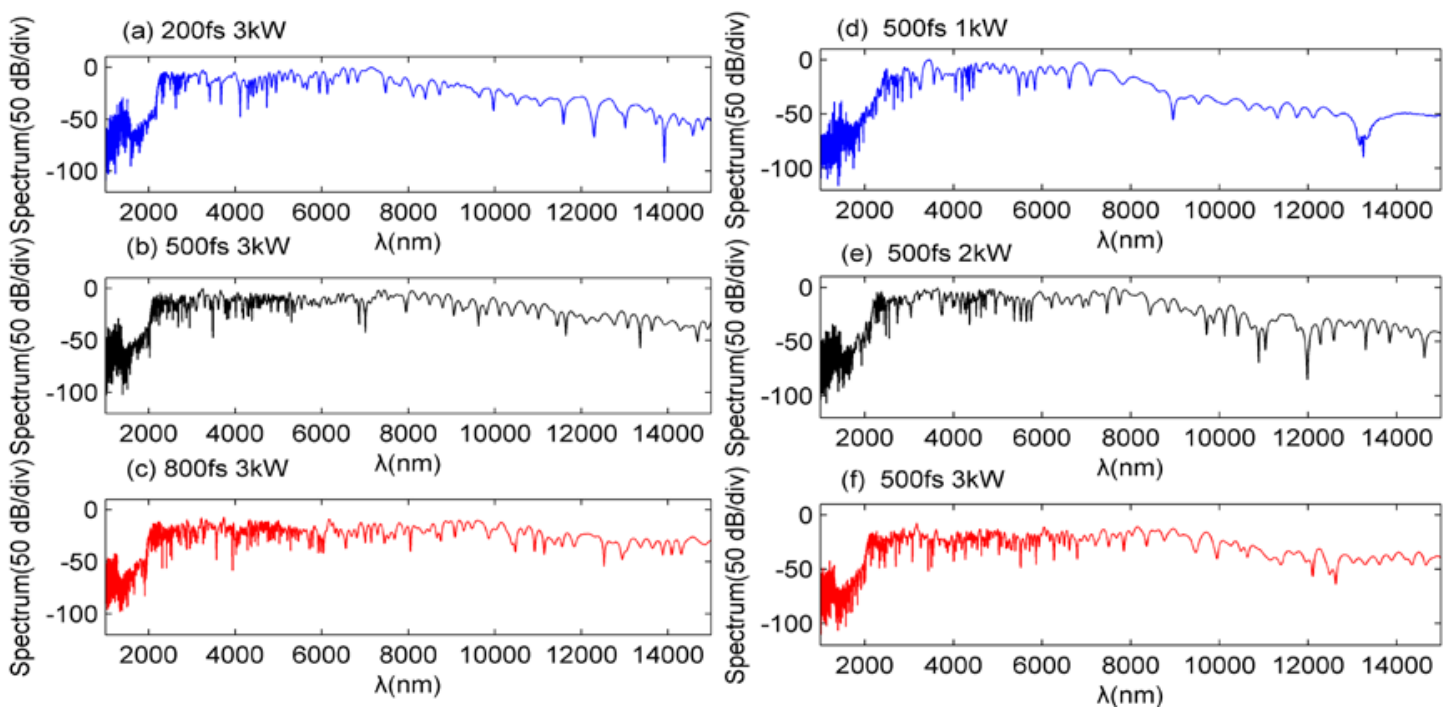

Fig. 4 The SC spectra of $\mathrm{As}_{2} \mathrm{Se}_{3}$ PCF pumped by a $4.3 \mu \mathrm{m}$ pulse with $3 \mathrm{~kW}$ peak power and pulse durations of (a) $200 \mathrm{fs}$, (b) $500 \mathrm{fs,} \mathrm{(c)} 800 \mathrm{fs}$, respectively; pulse with 500 pulse durations and peak power of (d) $1 \mathrm{~kW}$, (f) $2 \mathrm{~kW}$, (e) $3 \mathrm{~kW}$. 


\section{Conclusions}

Using As2Se3 PCF, the mechanism of MIR SC spectrum occurred. The influences of pulse width and peak power of input pulse on mid-infrared SC have been investigated. The results show that spectral broadening up to $15 \mu \mathrm{m}$ with a high spectral flatness is possible in As2Se3 PCF using a $4.3 \mu \mathrm{m}$ pump. And when the pump wavelength is near the ZDW, spectral broadening is biggest. Moreover, the pulse durations of the pump have positive influence on the spectrum broadening as well as the pump peak power. When the peak power is high, SC will more complex, spectrum will be further broadened.

\section{References}

1. J. M. Dudley and J. R. Taylor, “Ten years of nonlinear optics in photonic crystal fibre," Nat. Photon. 3(2), 85-90(2009).

2. R. Ho. Lzwarth, T. Udem, T. W. Hansch, "Optical frequency synthesizer for precision spectrocopy,” Phys. Rev. Lett. 85(11), 2264-2267(2000).

3. J. K. Ranka, R. S. Windeler, and A. J. Stentz, "Visible continuum generation in air-silica microstructure optical fibers with anomalous dispersion at 800 nm," Opt. Lett. 25(1), 25 - 27 (2000).

4. S. Roy, and P. R. Chaudhuri, "Supercontinuum generation in visible to mid-infrared region in square-lattice photonic crystal fiber made from highly nonlinear glasses," Opt. Commun. 282(17), 3448 - 3455(2009).

5. P. Domachuk, N. A. Wolchover, M. Cronin-Golomb, A. Wang, A. K. George, C. M. B. Cordeiro, J. C. Knight, F. G. Omenetto, “ Over 4000 nm Bandwidth of Mid-IR Supercontinuum Generation in sub-centimeter Segments of Highly Nonlinear Tellurite PCFs,” Opt. Express 16(10), 7161-7168(2008).

6. J. M. Dudley, G. Genty, S. Coen, “ Supercontinuum generation in photonic crystal fiber,” Rev. Mod. Phys. 78(4), 1135-1184(2006).

7. J. S. Sanghera, L. B. Shaw and I. D. Aggarwal, "Chalcogenide Glass-Fiber-Based Mid-IR Sources and Applications,” IEEE J. Sel. Top. Quantum Electron. 15(1), 114-119(2009).

8. G. Qin, X. Yan, C. Kito, M. Liao, C. Chaudhari, T. Suzuki and Y. Ohishi, "Ultrabroadband supercontinuum generation from ultraviolet to $6.28 \mu \mathrm{m}$ in a fluoride fiber," Appl. Phys. Lett. 95(16), 1103(2009).

9. J. Swiderski, M. Michalska and G. Maze, "Mid-IR supercontinuum generation in a ZBLAN fiber pumped by a gain-switched mode-locked Tm-doped fiber laser and amplifier system,” Opt. Express 21(7), 7851(2013).

10. Wu Yuan, “ $2-10 \mu \mathrm{m}$ mid-infrared supercontinuum generation in $\mathrm{As}_{2} \mathrm{Se}_{3}$ photonic crystal fiber, ”Laser Phys.Lett. 10(9), 095107(2013).

11. R. Cherif, A. B. Salem, M. Zghal et al., "Highly nonlinear $\mathrm{As}_{2} \mathrm{Se}_{3}$-based chalcogenide photonic crystal fiber for midinfrared supercontinuum generation,” Opt. Eng. 49(9), 095002(2010).

12. R. Cherif, M. Zghal, “ Ultrabroadband, Midinfrared Supercontinuum Generation in Dispersion Engineered $\mathrm{As}_{2} \mathrm{Se}_{3}$-Based Chalcogenide Photonic Crystal Fibers,” I. J. Opt. 70(4), 876474(2013).

13. L. B. Shaw, V. Q. Nguyen, J. S. Sanghera, I. D. Aggarwal, P. A.Thielen, and F. H. Kung, "IR supercontinuum generation in As-Se photonic crystal fiber,” ASSP. TuC5, 864 - 868(2005).

14. J. H. V. Price, T. M. Monro, H. Ebendorff-Heidepriem, "Mid-IR supercontinuum generation from nonsilica microstructured optical fibers,” IEEE J. Sel. Top. Quantum Electron. 13(3), 738 - 749(2007).

15. X. Y. Wang, S. G. Li, S. Liu, G. B. Yin, J. S. Li, "Generation of a mid-infrared broadband polarized supercontinuum in $\mathrm{As}_{2} \mathrm{Se}_{3}$ photonic crystal fibers," Chin. Phys. B. 21(5), 
054220(2012). 